\title{
DYNAMICS OF ACCRETION FLOWS IRRADIATED BY A QUASAR
}

\author{
DANIEL Proga ${ }^{1}$ \\ ${ }^{1}$ Department of Physics, University of Nevada, Las Vegas, NV 89154, USA, e-mail: dproga@physics.unlv.edu \\ Draft version September 28, 2018
}

\begin{abstract}
We present the results from axisymmetric time-dependent hydrodynamical calculations of gas flows which are under the influence of gravity of a black hole in quasars. We assume that the flows are nonrotating and exposed to quasar radiation. We take into account X-ray heating and the radiation force due to electron scattering and spectral lines. To compute the radiation field, we consider an optically thick, geometrically thin, standard accretion disk as a source of UV photons and a spherical central object as a source of X-rays. The gas temperature and ionization state in the flow are calculated self-consistently from the photoionization and heating rate of the central object.

We find that for a $10^{8} \mathrm{M}_{\odot}$ black hole with an accretion luminosity of 0.6 of the Eddington luminosity the flow settles into a steady state and has two components: (1) an equatorial inflow and (2) a bipolar inflow/outflow with the outflow leaving the system along the disk rotational axis. The inflow is a realization of a Bondi-like accretion flow. The second component is an example of a non-radial accretion flow which becomes an outflow once it is pushed close to the rotational axis where thermal expansion and the radiation pressure accelerate it outward. Our main result is that the existence of the above two flow components is robust to the outer boundary conditions and the geometry and spectral energy distribution of the radiation field. However, the flow properties are not robust. In particular, the outflow power and collimation is higher for the radiation dominated by the UV/disk emission than for the radiation dominated by the X-ray/central engine emission. Our most intriguing result is that a very narrow outflow driven by radiation pressure on lines can carry more energy and mass than a broad outflow driven by thermal expansion.
\end{abstract}

Subject headings: accretion, accretion disks - methods: numerical - HD

\section{INTRODUCTION}

Active Galactic Nuclei (AGN) in general, but quasars in particular are very powerful sources of radiation. The AGN luminosity, $L$ is typically high compared to its Eddington limit, $L_{\text {Edd }}\left(0.001 L_{\text {Edd }} \lesssim L \lesssim 1 L_{\text {Edd }}\right)$. The spectral energy distribution (SED) of AGN is very broad. It spans the wavelength range from radio to hard $\mathrm{X}$-rays and even TeV. Most of the AGN luminosity is in the opticalUV-IR bands but some significant fraction is in the X-ray band.

These AGN radiation properties and the AGN central location in their host galaxies imply that they play a very important role in determining the ionization structure and dynamics of matter not only in their vicinity but also on larger, galactic and even intergalactic scales (Ciotti \& Ostriker, 1997, 2001; King 2003; Murray, Quataert, \& Thompson 2005; Sazonov et al. 2005; Springel, Di Matteo \& Hernquist 2005; Hopkins et al. 2005; Wang, Chen, \& $\mathrm{Hu}$ and references therein). There are many indications that support this suggestion, for example the presence of broad emission lines (BELs) in AGN spectra.

BELs are one of the defining spectral features of AGN. They are observed in optical (OPT) and ultraviolet (UV) spectra and have line wings extending to velocities up to $10^{4} \mathrm{~km} \mathrm{~s}^{-1}$. It is fairly well established that the primary physical mechanism for production of BELs is photoionization by the compact continuum source of AGN. Detailed photoionization calculations yield relatively tight constraints on the physical conditions of the emitting gas - such as the temperature, density, ionization level, and chemical abundances (e.g., Ferland et al. 1998; Hamman \& Ferland 1999; Krolik 1999 and references therein). The width of BELs indicates that the photoionized gas is supersonic. The shape and position of the line profiles can be explained by lines forming either in a region without preferred velocity direction and with a nearly spherical distribution or at the base of a wind from an accretion disk (Murray et al. 1995, hereafter MCGV; Bottorff et. al 1997). In the latter case, the BELs can form where the outflow expansion velocity is low compared to the rotational velocity (MCGV).

Not all gas in AGN shares the properties of the one responsible for BELs. Some quasars show broad absorption lines (BALs) which are the most dramatic evidence for well-organized outflows in AGN. BALs are almost always blueshifted relative to the emission-line rest frame, indicating the presence of outflows from the active nucleus, with velocities as large as 0.2 c (e.g., Turnshek 1998). BALs are observed not only in the UV but also in the X-rays. For example, Chartas, Brandt \& Gallagher (2003) discovered a very broad absorption line in the X-ray spectrum of PG 1115+80. Other evidence for AGN outflows include narrow absorption lines (NALs). UV spectra of some quasars show NALs which are blueshifted by as much as $\sim 50000 \mathrm{~km} \mathrm{~s}^{-1}$ (Hamann et al. 1997). NALs are found much more commonly in the UV spectra of Seyfert galaxies than of quasars but in Seyfert galaxies the lines are blueshifted only by several $100 \mathrm{~km} \mathrm{~s}^{-1}$ (Crenshaw et al. 1999). As BALs, NALs are observed not only in the UV but also in the X-rays. For example, Kaastra et al. 
(2000) observed NALs due to highly ionized species in a high-resolution X-ray observation of the Seyfert galaxy NGC 5548 obtained by Chandra. As mentioned above, some argue that BELs may also be associated with the base of a disk outflow.

The dynamics of the gas responsible for BALs, BELs, and NALs can be driven by radiation, even for subEddington sources. The driving can be due to radiation pressure or radiation heating, or both. The radiation force can overcome gravity for sub-Eddington sources when the gas opacity is higher than the electron scattering. The latter is usually used to define $L_{\mathrm{Edd}}$. The gas opacity can be enhanced by scattering of photons by UV spectral lines. Radiation pressure on spectral lines (line force) can be significant, provided gas is moderately ionized and can interact with the UV continuum through very many UV line transitions. For highly ionized gas, line force is negligible because of a lower concentration of ions capable of providing UV line opacity. In the case of highly or fully ionized gas, an outflow still can be produced if the gas heating is efficient enough for the thermal energy to exceed the gravitational energy. AGN with their broad SED, are systems where both line driving and radiation heating, in particular, X-ray heating can operate.

The gas dynamics can be also affected by dust. For example radiation pressure due to the dust opacity can produce an outflow of dust and also of gas, as the two can be coupled (e.g., Phinney 1989; Pier \& Krolik 1992; Emmering, Blandford, \& Shlosman 1992; Laor \& Draine 1993; Königl \& Kartje 1994; Murray et al. 2005 and references therein). Matter in AGN is known to be a mixture of gas and dust on large scales (e.g., Miller \& Goodrich 1985; Awaki et al. 1991; Antonucci 1984; Blanco, Ward, \& Wright 1990; Krolik 1999 and references therein) but also on subparsec scales as in the Seyfert galaxy NGC 1068 (e.g., Wittkowski et al. 2004). However, details of the matter composition and microphysics are very complex and poorly understood. Additionally, the presence of dust affects the state and dynamics of gas and vice verse. In particular, it is nontrivial to include the dust effects on the gas photoionization state. Therefore studies that consider both matter components rely on simplifying assumptions regarding the dynamics and geometry (e.g., Murray, Quataert, \& Thompson 2005). There are also studies that focus only on the gas component and consider dynamics on relatively smaller radii (e.g., Sazonov et al. 2005). We follow the latter and focus on the gas dynamics.

It is commonly accepted that AGN are powered by disk accretion of matter onto a supermassive black hole $(\mathrm{BH})$. A wind driven from such a disk by line force is the most promising hydrodynamical (HD) scenario for AGN outflows, especially high luminsity quasars. In this scenario, a wind is launched from the disk by the local disk radiation at radii where the disk radiation is mostly in the UV (Shlosman, Vitello \& Shaviv 1985; MCGV). Such a wind is continuous and has mass loss rate and velocity which are capable of explaining the blueshifted absorption lines observed in many AGN, if the ionization state is suitable (e.g., MCGV, Proga, Stone \& Kallman 2000, PSK hereafter; Proga \& Kallman 2004). This wind scenario has the desirable feature that for the wind motive power, it relies on radiation which is an observable quantity. However, not all AGN outflows can be explained by line driving be- cause of too low luminosity or too high ionization state or both (e.g., Chelouche \& Netzer 2005; and Kraemer et al. 2006). Therefore, other mechanisms such as thermal and magnetic driving are likely important.

Theoretical models predict that X-ray heating can have profound effects on the gas dynamics in disks. Since X-rays tend to heat low density gas to a temperature $T_{\mathrm{C}} \sim 10^{7} \mathrm{~K}$, with which matter in an accretion disk is expected to either puff up and form a static corona, or to produce a thermal wind, depending on whether the thermal velocity exceeds the local escape velocity, $v_{\text {esc }}$ (e.g. Begelman, McKee and Shields, 1982; Ostriker, McKee, \& Klein 1991; Woods et al. 1996; Proga \& Kallman 2002). This paper, and this section in particular, are focused on non-magnetic processes. Magnetic driving is briefly discussed in $\S 4$ (see also Proga 2007).

The insights gained from these studies support the radiation driven disk wind model for outflows in quasars and likely in other AGN. These successes motivate further exploration of radiation effects on gas dynamics in and outside AGN using similar methods. The particular issues needed attention include: (1) Can AGN radiation drive an outflow from anywhere else than an accretion disk (e.g., from a large scale torus believed to surround an AGN or from an inflowing gas accreting directly onto a $\mathrm{BH}$ or indirectly through an accretion disk)? (2) If so, what is the power of such an outflow and can AGN radiation control the rate at which matter is supplied to the AGN accretion disk. More broadly, is the AGN radiation an important element of the so-called AGN feedback? (3) What is the origin and the dynamics of matter responsible for NALs and NELs?

We report here on results from our first phase of studying gas dynamics in AGN on sub- and parsec-scales. We have simplified several aspects of this complex problem. For example, we have not taken into account rotation of the flow and the magnetic fields. Additionally, we have not taken into account dust as we assume that all the dust has been destroyed. This assumption is valid interior to the dust sublimation radius, where the central object radiation is responsible for the dust destruction. At large radii, still inside our computational domain, this assumption is less valid but the dust could be destroyed there by other processes such as dust sputtering. Our goal is to set-up simulations as simple as possible to study the effects of the X-ray heating (important in the so-called preheated accretion, see Ostriker et al. 1976) and radiation pressure on gas which is initially captured by a $\mathrm{BH}$.

We decided to study non-rotating gas because once this gas is initially located in the polar region at large radii, it will likely stay in this region regardless of the subsequently radial movement with respect to the $\mathrm{BH}$ and its accretion disk, i.e, it will not settle down on the accretion disk. Therefore non-rotating gas can be exposed to the direct disk radiation for a wide range of radii and radial velocities. For these reasons, non-rotating gas can easily absorb, re-emit, and scatter the disk radiation and be responsible for line emission and absorption in AGN.

We use the methods developed by PSK to study gas dynamics on sub-parsec- and parsec-scales in AGNs. We consider an axisymmetric HD flow accreting onto a supermassive $\mathrm{BH}$. The flow is non-spherical because it is irradiated by an accretion disk and spherical corona. The disk 
radiation flux is the highest along the disk rotational axis and is gradually decreasing with increasing polar angle, $\theta$ as $\cos \theta$. The corona radiation is isotropic. We take into account the radiation heating and cooling, radiation pressure due to the electron scattering and spectral lines. We adopt a simplified treatment of photoionization, and radiative cooling and heating that allow us to compute self-consistently the ionization state, and therefore the line force, in the flow.

We concentrate on assessing what fraction of the inflow from large radii reaches the vicinity of a $\mathrm{BH}$ and what fraction is turned into an outflow. We also assess the impact of thermal expansion and radiation pressure on driving the outflow and on the outflow properties such as the two-dimensional structure, thermal and kinetic power and temporal behavior. We describe our calculation in Section 2. We present our results in Section 3. The paper ends, in section 4, with discussion and our conclusions.

\section{METHOD}

\subsection{Hydrodynamics}

To compute the structure and evolution of a flow irradiated by an AGN, we solve the equations of hydrodynamics

$$
\begin{gathered}
\frac{D \rho}{D t}+\rho \nabla \cdot \mathbf{v}=0, \\
\rho \frac{D \mathbf{v}}{D t}=-\nabla P+\rho \mathbf{g}+\rho \mathbf{F}^{\mathrm{rad}} \\
\rho \frac{D}{D t}\left(\frac{e}{\rho}\right)=-p \nabla \cdot \mathbf{v}+\rho \mathcal{L},
\end{gathered}
$$

where $\rho$ is the mass density, $P$ is the gas pressure, $\mathbf{v}$ is the velocity, $e$ is the internal energy density, $\mathcal{L}$ is the net cooling rate, $\mathbf{g}$ is the gravitational acceleration of the central object, $\mathbf{F}^{\mathrm{rad}}$ is the total radiation force per unit mass. We adopt an adiabatic equation of state $P=(\gamma-1) e$, and consider models with the adiabatic index, $\gamma=5 / 3$.

To solve eqs. 1-3, we use the ZEUS-2D code (Stone \& Norman 1992) extended by PSK. We perform our calculations in spherical polar coordinates $(r, \theta, \phi)$ assuming axial symmetry about the rotational axis of the accretion disk $\left(\theta=0^{\circ}\right)$.

Our standard computational domain is defined to occupy the angular range $0^{\circ} \leq \theta \leq 90^{\circ}$ and the radial range $r_{\mathrm{i}}=500 r_{*} \leq r \leq r_{\mathrm{o}}=2.5 \times 10^{5} r_{*}$, where $r_{*}=3 r_{\mathrm{S}}$ is the inner radius of the disk around a Schwarzschild $\mathrm{BH}$ with a mass, $M_{\mathrm{BH}}$ and radius $r_{\mathrm{S}}=2 G M_{\mathrm{BH}} / c^{2}$. The $r-\theta$ domain is discretized into zones. For $r_{\mathrm{o}}=2.5 \times 10^{5} r_{*}$ and $1 \times 10^{6} r_{*}$, our numerical resolution in the $r$ direction consists of 140 and 180 zones, respectively. We fix the zone size ratio, $d r_{k+1} / d r_{k}=1.04$ (i.e., the zone spacing is increasing outward). Gridding in this manner ensures good spatial resolution close to the inner boundary at $r_{\mathrm{i}}$. In the $\theta$ direction, our standard numerical resolution consists of 50 but we also performed simulations with 100 zones. Zone size ratio is always $d \theta_{l} / d \theta_{l+1}=1.0$ (i.e., grid points are equally spaced).

For the initial condition, we typically assume spherical symmetry set all HD variables to constant values everywhere in the computational domain, i.e., $\rho(r, \theta)=\rho_{0}$ $e(r, \theta)=e_{0}, v_{r}(r, \theta)=v_{r, 0}=0, v_{\theta}(r, \theta)=v_{\theta, 0}=0$, and $v_{\phi}(r, \theta)=v_{\phi, 0}=0$. For comparison, we performed simulations where we initially set $\mathrm{HD}$ variables to values which resulted from one-dimensional simulations without radiation pressure.

We specify the boundary conditions in the following way. At the pole (i.e., $\theta=0^{\circ}$ ), we apply an axis-of-symmetry boundary condition while for $\theta=90^{\circ}$, we apply reflecting boundary conditions. For the inner and outer radial boundaries, we apply an outflow boundary condition (i.e., to extrapolate the flow beyond the boundary, we set values of variables in the ghost zones equal to the values in the corresponding active zones, see Stone \& Norman 1992 for more details). To represent steady conditions at the outer radial boundary, during the evolution of each model we continue to apply the constraints that in the last zone in the radial direction, $v_{\theta}\left(r_{\mathrm{o}}, \theta\right)=v_{\phi}\left(r_{\mathrm{o}}, \theta\right)=0$, and the density and internal energy are fixed at constant values at all times. We allow $v_{r}$ to float. We have found that this technique produces a solution that relaxes to a steady state solution for both spherically symmetric accretion and non spherical accretion with an outflow (see also Proga \& Begelman 2003a). Our approach then is to mimic the situation where there is always gas available for accretion.

\subsection{The radiation field and force}

The geometry and assumptions needed to compute the radiation field from the disk and central object are similar to those considered by PSK but here we make some simplifications to the earlier model. The disk is flat, Keplerian, geometrically-thin and optically-thick. The disk photosphere is coincident with the $\theta=90^{\circ}$ axis. We specify the radiation field of the disk by assuming that the temperature follows the radial profile of the optically thick accretion disk (Shakura \& Sunyaev 1973), and therefore depends on the mass accretion rate in the disk, $\dot{M}_{\mathrm{D}}$, the mass of the $\mathrm{BH}, M_{\mathrm{BH}}$ and the inner edge of the disk, $r_{*}$, In particular, the total accretion luminosity, $L=2 \eta G M_{\mathrm{BH}} \dot{M}_{\mathrm{a}} / r_{\mathrm{S}}$, where $\eta$ is the rest mass conversion efficiency.

The geometry of the central engine in AGNs is poorly known. As in PSK, we consider the central engine as the most inner part of the accretion disk plus an extended corona. We refer to the corona as the central object. The inner radius of the computational domain is large compared to the radius of the central object. Therefore the central object can be approximated as a point source located at $r=0$. We express the disk luminosity, $L_{\mathrm{D}}$ and the central object luminosity, $L_{*}$ in units of the total accretion luminosity, i.e., $L_{\mathrm{D}}=f_{\mathrm{D}} L$ and $L_{*}=f_{*} L=\left(1-f_{\mathrm{D}}\right) L$. For simplicity, we assume that the disk emitts only in the UV, whereas the central object emitts only the X-rays. i.e., the system UV luminosity, $L_{\mathrm{UV}}=f_{\mathrm{UV}} L=L_{\mathrm{D}}$ and the system X-ray luminosity, $L_{\mathrm{X}}=f_{\mathrm{X}} L=L_{*}$ (in other words $f_{\mathrm{UV}}=f_{\mathrm{D}}$ and $f_{\mathrm{X}}=f_{*}$ )

With the above simplifications, only the central object radiation is responsible for ionizing the flow to a very high state. The central radiation contributes to the radiation force due to electron scattering but does not contribute to line driving. On the other hand, the disk radiation contribute to the radiation force due to both electrons and lines. Note that we assume that the luminosity in the remaining bands, mainly optical and infrared, is the part of 
the luminosity that does not change the dynamics of the wind and set it to zero.

To evaluate, line force, we generally follow PSK who used a modified Castor, Abbott \& Klein method (Castor, Abbott, \& Klein 1975; see also Proga, Stone, Drew 1998, 1999). The line force at a point defined by the position vector $\mathbf{r}$ is

$$
\mathbf{F}^{\mathrm{rad}, 1}(\mathbf{r})=\oint_{\Omega} M(t)\left(\hat{n} \frac{\sigma_{e} I(\mathbf{r}, \hat{n}) d \Omega}{c}\right)
$$

where $I$ is the frequency-integrated continuum intensity in the direction defined by the unit vector $\hat{n}$, and $\Omega$ is the solid angle subtended by the disk and central object at the point. The term in brackets is the electron-scattering radiation force, $\sigma_{e}$ is the mass-scattering coefficient for free electrons, and $M(t)$ is the force multiplier (Castor et al. 1975 ) - the numerical factor which parametrizes by how much spectral lines increase the scattering coefficient. In the Sobolev approximation, $M(t)$ is a function of the optical depth parameter

$$
t=\frac{\sigma_{e} \rho v_{\mathrm{th}}}{\left|d v_{l} / d l\right|}
$$

where $v_{\text {th }}$ is the thermal velocity, and $\frac{d v_{l}}{d l}$ is the velocity gradient along the line of sight, $\hat{n}$.

Evaluation of the radiation force due to an nonisothermal extended source such as an accretion disk is relatively expensive (see PSK, Proga et al. 1999). However, we consider here the flow relatively far from both the central object and the most luminous part of the disk. Therefore, we simplify the force evaluation as follows. First, we consider only the radial component of the force, $\mathbf{F}_{r}^{\mathrm{rad}, 1}$ and set the other components to zero. Second, we compute the velocity gradient by taking into account only the dominant term: gradient of the radial velocity along the radial direction, i.e. $\frac{d v_{r}}{d r}$.

With these simplifications and an assumption that the flow does not change the geometry of the radiation field, we can evaluate the radiation force in two steps: (1) calculation of the velocity gradient in the $\hat{n}_{r}$ direction and then the optical depth parameter $t$; (2) calculation of the parameters of the force multiplier using a current value of the photoionization parameter, $\xi$ adopting results of Stevens \& Kallman (1990). Then we calculate the radiation force exerted by radiation along the radial direction. Our approach to evaluate the radiation forces is simplified compared to the approach used in PSK because we do not integrate the radiation force over the solid angle subtended by the radiant surface but instead use an analytic approximation (compare eqs. 4 and 8), and we do not correct the radiation force for the optical depth effects.

Below we describe in more detail, our calculations of the force multiplier and the radiation force for various conditions in the flow. For $r \gg r_{*}$, in the optically thin case, the radial radiation flux from a disk and central object can be written as

$$
\mathcal{F}_{\mathrm{D}}(r, \theta)=2 \cos \theta \frac{L_{\mathrm{D}}}{4 \pi r^{2}}
$$

and

$$
\mathcal{F}_{*}(r)=\frac{L_{*}}{4 \pi r^{2}},
$$

respectively. Eq. 6 approximates the numerical evaluation of the radiation flux integral over the solid angle subtended by the disk surface (see eqs B2 and B3 in Proga, Stone \& Drew 1998) while eq. 7 is the exact solution for the radiation flux for a point source. With eqs. 6 and 7 , one can show that eq. 4 reduces to

$$
\mathbf{F}_{r}^{\mathrm{rad}}(r, \theta)=\frac{\sigma_{e} L}{4 \pi r^{2} c}\left[f_{*}+2 \cos \theta f_{\mathrm{D}}(1+M(t))\right]
$$

To compute the force multiplier, we adopt Castor et al.'s (1975) analytical formula modified by Owocki, Castor \& Rybicki (1988 see also Proga et al. 1998)

$$
M(t)=k t^{-\alpha}\left[\frac{\left(1+\tau_{\max }\right)^{(1-\alpha)}-1}{\tau_{\max }^{(1-\alpha)}}\right]
$$

where $k$ is proportional to the total number of lines, $\alpha$ is the ratio of optically-thick to optically-thin lines, $\tau_{\max }=t \eta_{\max }$ and $\eta_{\max }$ is a parameter determining the maximum value, $M_{\max }$ achieved for the force multiplier. Equation 9 shows the following limiting behavior:

$$
\begin{aligned}
& \lim _{\tau_{\max \rightarrow \infty} M(t)}=k t^{-\alpha} \\
& \lim _{\tau_{\max } \rightarrow 0} M(t)=M_{\max },
\end{aligned}
$$

where $M_{\max }=k(1-\alpha) \eta_{\max }^{\alpha}$.

To compute, the parameters of $M(t)$ taking into account the effects of ionization, we follow PSK. In particular, we compute the parameters of the line force using a value of the photoionization parameter, $\xi$ and the analytical formulae for $k$ and $\eta_{\max }$ from Stevens \& Kallman (1990):

$$
k=0.03+0.385 \exp \left(-1.4 \xi^{0.6}\right),
$$

and

$$
\log _{10} \eta_{\max }= \begin{cases}6.9 \exp \left(0.16 \xi^{0.4}\right) & \text { for } \log _{10} \xi \leq 0.5 \\ 9.1 \exp \left(-7.96 \times 10^{-3} \xi\right) & \text { for } \quad \log _{10} \xi>0.5\end{cases}
$$

The parameter $\alpha=0.6$ and does not change with $\xi$.

Our procedure to calculate $M(t)$ is computationally efficient and gives approximate estimates for the number and opacity distribution of spectral lines for a given $\xi$ without detail information about the ionization state. However, it is just an estimate because Stevens \& Kallman (1990) results were computed for the case where a $25000 \mathrm{~K}$ stellar atmosphere was irradiated by a $10 \mathrm{keV}$ bremsstrahlung ionizing spectrum whereas we consider gas not of a stellar atmosphere and its temperature, without irradiation, depends of the outer boundary conditions. Additionally, the AGN spectrum is different from a bremsstrahlung spectrum. As we describe below, the parameters $k$ depends on the gas temperature (i.e., eq. 17). Additionally this and other parameters of $M(t)$ depend also on the SED of the ionizing and UV radiation. In particular, $\log _{10} \xi=-0.5$ and the UV emission caused by the low energy extension of the $10 \mathrm{keV}$ bremsstrahlung, $M_{\max } \approx 300$ whereas for an AGN-like UV spectrum, i.e., a power law with the energy index equal to $1, M_{\max } \approx 8000$ (T. Kallman private communication). For comparison, eqs. 12 and 13 yield $M_{\max } \approx 3400$ also for $\log _{10} \xi=-0.5$. A detail investigation of these and other dependencies is beyond the scope 
of this study but it is important to carry out such a study in the future.

To calculate the photoionization parameter needed in eqs. 12 and 13 and in evaluation of the net radiative cooling, we use the formula:

$$
\xi=\frac{4 \pi \mathcal{F}_{\mathrm{X}}}{n},
$$

where $\mathcal{F}_{\mathrm{X}}$ is the local $\mathrm{X}$-ray flux, $n$ is the number density of the gas $\left(=\rho /\left(m_{p} \mu\right)\right.$, where $m_{p}$ is the proton mass, and $\mu$ is the mean molecular weight). We consider models with $\mu=1$. The local X-ray flux is corrected for the optical depth effects:

$$
\mathcal{F}_{\mathrm{X}}=\mathcal{F}_{*} \exp \left(-\tau_{\mathrm{X}}\right),
$$

where $\tau_{\mathrm{X}}$ is the X-ray optical depth. We estimate $\tau_{\mathrm{X}}$ between the central source and a point in a flow from:

$$
\tau_{\mathrm{X}}=\int_{0}^{r} \kappa_{\mathrm{X}} \rho d r
$$

where $\kappa_{\mathrm{X}}$ is the absorption coefficient, and $r$ is the distance from the central source.

The flow physical conditions and consequently the line force depend not only on $\xi$. In particular, the gas temperature and ionization state can be affected by adiabatic heating or cooling. For example, the line force can be neglected even in the region of low $\xi$ if this region is hot due to adiabatic compression. Therefore, we first compute the line force parameters based on $\xi$ and then correct the parameters for the gas temperature effects.

Specifically, we compute the parameter $k$ using the expression

$\log _{10} k= \begin{cases}-0.383 & \text { for } \log _{10} T \leq 4 \\ -0.630 \log _{10} T+2.138 & \text { for } 4<\log _{10} T \leq 4.75 \\ -3.870 \log _{10} T+17.528 & \text { for } \log _{10} T>4.75\end{cases}$

We adopt this value of $k$ if it is smaller than the one obtained by using expression 12 . Expression 17 is based on detailed photoionization calculations performed using the XSTAR code (T. Kallman private communication).

These expressions for the parameters of the force multiplier predict that $M_{\max }$ increases gradually from $\sim 2000$ to 5000 as $\xi$ increases from 0 to $\sim 3$ and then drops to $\sim 0.1$ at $\xi=1000$. The line force becomes negligible for $\xi \gtrsim 100$ because then $M_{\max } \sim 1$. The line force becomes also negligible if $T>10^{5} \mathrm{~K}$ for any $\xi$.

\subsection{Radiation heating and cooling}

To calculate the gas temperature we also follow PSK, who assumed that the gas is optically thin to its own cooling radiation. The net cooling rate depends on the density, $\rho$, the temperature, $T$, the ionization parameter $\xi$, and the characteristic temperature of the X-ray radiation $T_{\mathrm{X}}$. We refer a reader to PSK and Proga \& Kallman (2004) for more details.

We calculate the evolution of the internal energy and therefore we specify the initial and outer boundary conditions for $e$. We do this in the following way: we specify the gas temperature at the outer boundary (in the last grid zone in the radial direction), $T_{0}$, by setting it to the
Compton temperature, $T_{\mathrm{C}}=0.25 T_{\mathrm{X}}$. Then we calculate the internal energy from

$$
e_{0}=\frac{\rho_{\mathrm{o}} k T_{0}}{\mu m_{p}(\gamma-1)}
$$

\section{RESULTS}

We assume the mass of the non-rotating $\mathrm{BH}$, $M_{\mathrm{BH}}=10^{8} \mathrm{M}_{\odot}$ and the disk inner radius, $r_{*}=3 r_{\mathrm{S}}=8.8 \times 10^{13} \mathrm{~cm}$ throughout this paper. We consider the rest mass conversion efficiency $\eta=0.0833$. We set $\dot{M}_{\mathrm{a}}$ to be $10^{26} \mathrm{~g} \mathrm{~s}^{-1}\left(=1.6 \mathrm{M}_{\odot} \mathrm{yr}^{-1}\right)$. These system parameters yield the accretion luminosity, $L=7.5 \times 10^{45} \mathrm{erg} \mathrm{s}^{-1}\left(=2 \times 10^{12} \mathrm{~L}_{\odot}\right)$. This luminosity corresponds to the Eddington number, $\Gamma=0.6$. We define the Eddington number as $L$ in units of the Eddington luminosity for the Schwarzschild BH, $L_{\mathrm{Edd}}=4 \pi c G M_{\mathrm{BH}} / \sigma_{e}$ [i.e., $\left.\Gamma \equiv L / L_{\mathrm{Edd}}=\left(\sigma_{e} \dot{M}_{\mathrm{a}}\right) /\left(8 \pi c r_{\mathrm{S}}\right)\right]$. To determine the radiation field, we specify also $f_{\mathrm{UV}}$ and $f_{\mathrm{X}}$. We note that we fix $\dot{M}_{\mathrm{a}}$ during our simulations, i.e. $\dot{M}_{\mathrm{a}}$ does not depend on the mass flux through the inner boundary, $\dot{M}_{\text {in }}\left(r_{\mathrm{i}}\right)$ (see eq. 20 for the formal definition of $\dot{M}_{\text {in }}\left(r_{\mathrm{i}}\right)$ and $\S 4$ for discussion of this aspect of the calculations).

The parameters $f_{\mathrm{UV}}$ and $f_{\mathrm{X}}$ (and also $f_{\mathrm{D}}$ and $f_{*}$ ) specifying the radiation field are our free parameters which we focus on exploring the most in this paper. We consider three cases: case A with $f_{\mathrm{UV}}=0.5$ and $f_{\mathrm{X}}=0.5$, case $\mathrm{B}$ with $f_{\mathrm{UV}}=0.8$ and $f_{\mathrm{X}}=0.2$, and case $\mathrm{C}$ with $f_{\mathrm{UV}}=0.95$ and $f_{\mathrm{X}}=0.05$ (see Table 1 for summary of our runs). The spectral energy distribution of the ionizing radiation is not well known, our choice of values for $f_{\mathrm{UV}}$ and $f_{\mathrm{X}}$ is guided by the observational results from Zheng et al. (1997) and Laor et al. (1997).

To calculate the gas temperature, we assume the temperature of the X-ray radiation, $T_{\mathrm{X}}=8 \times 10^{7} \mathrm{~K}$ (e.g., Sazonov, Ostriker \& Sunyaev 2005 and references therein) and the line cooling parameter $\delta=1$ (see Blondin 1994 and PSK). The force multiplier depends only formally on the thermal speed, $v_{\mathrm{th}}$. Therefore to compute $t$, we set to $20 \mathrm{~km} \mathrm{~s}^{-1}$, i.e., the thermal speed of a hydrogen atom at the temperature of $25000 \mathrm{~K}$ for which the parameters of the force multiplier were computed (Stevens \& Kallman 1990). Finally, the attenuation of the X-rays is calculated using $\kappa_{\mathrm{X}}=0.4 \mathrm{~g}^{-1} \mathrm{~cm}^{2}$ for all $\xi$. The resulting optical depth corresponds to the Thomson optical depth.

For our parameters, both the so-called Compton radius, $R_{\mathrm{C}} \equiv G M_{\mathrm{BH}} \mu m_{p} / k T_{\mathrm{C}}=8 \times 10^{18} \mathrm{~cm}=9 \times 10^{4} r_{*}$ and the Bondi radius, $R_{\mathrm{B}}=G M_{\mathrm{BH}} / c_{\infty}^{2}=4.8 \times 10^{18} \mathrm{~cm}=$ $5.5 \times 10^{4} r_{*}$ (Bondi 1952) are inside our computational domain. We assumed here that the gas temperature at infinity $T_{\infty}=T_{\mathrm{C}}=2 \times 10^{7} \mathrm{~K}$ so that the sound speed at infinity, $c_{\infty}=\left(\gamma k T_{\mathrm{C}} / \mu m_{p}\right)^{1 / 2}=4 \times 10^{7} \mathrm{~cm} \mathrm{~s}^{-1}$ and $R_{\mathrm{C}}=$ $\gamma R_{\mathrm{B}}$. For the isothermal flow, the Bondi accretion rate is $\dot{M}_{\mathrm{B}}=3.3 \times 10^{25} \mathrm{~g} \mathrm{~s}^{-1}=0.52 \mathrm{M}_{\odot} \mathrm{yr}^{-1}$. We note that the dust sublimation radius, $R_{\text {sub }}=2.6 \times 10^{18} \mathrm{~cm}=3 \times 10^{4} r_{*}$ (e.g., Murray et al. 2005, eq. 41) is also inside the domain.

We consider the situation where the BH gravity is reduced by the $\theta$-dependent radiation force. Therefore, the effective gravity is also $\theta$ dependent. Using eqs. 6 and 7 , the location where thermal energy equals the effect gravitational energy (i.e., a modified Compton radius) can be 
written as

$$
\bar{R}_{\mathrm{C}} \equiv R_{\mathrm{C}}\left[1-\Gamma\left(2 \cos \theta f_{\mathrm{D}}+f_{*}\right)\right] .
$$

Figure 1 presents the results for cases A, B, and $\mathrm{C}$ with $\rho_{0}=10^{-21} \mathrm{~g} \mathrm{~cm}^{-3}, v_{r, 0}=v_{\theta, 0}=v_{\phi, 0}=0$. At the outer radial boundary, during the evolution of each model we continue to apply the constraints that the density and internal energy (the latter is computed for $T_{0}=T_{\mathrm{C}}$ ) are fixed at constant values at all times. The figure shows the instantaneous density and temperature distributions, and the poloidal velocity field of the models. Additionally, it shows $\bar{R}_{\mathrm{C}}$ and the contours of the Mach number , $M \equiv \sqrt{v_{r}^{2}+v_{\theta}^{2}} / c_{s}$, where $c_{s}=\gamma P / \rho$ is the sound speed. For all three cases, the flow settles quickly into a steady state (within $\sim$ a few $\times 10^{12}$ s which correspond to a few dynamical time scales at the outer radius, $\left.\tau=\left(r_{o}^{3} / G M_{\mathrm{BH}}\right)^{1 / 2}=9 \times 10^{11} \mathrm{~s}\right)$. The steady state consists of two flow components (1) an equatorial inflow and (2) a bipolar inflow/outflow with the outflow leaving the system along the pole. The outflow is collimated by the infalling gas. Our calculations capture the subsonic and supersonic parts of both the inflow and outflow. Although the same components can be identified in the three cases their size, density and temperature, and the degree of outflow collimation depend on the SED and the geometry of the radiation field.

In run A, X-ray heating is the strongest and it accelerates an outflow to the maximum velocity of $700 \mathrm{~km} \mathrm{~s}^{-1}$. Although, the gas temperature decreases in the inflow down to some radius, it is not low enough for line force to be significant. The outflow collimation by the infall increases with increasing radius. This tendency is clearly seen in the other two cases.

For run B, the outflow is strongly pushed toward the pole at $r \approx 1.2 \times 10^{5} r_{*}$ and the flow pattern resembles a bottle neck where the density increases by a couple of orders of magnitude. The gas temperature drops there to the level below $10^{5} \mathrm{~K}$. In this low temperature region line driving accelerates an outflow to the velocity up to $4000 \mathrm{~km} \mathrm{~s}^{-1}$ which is significantly higher than the velocity of the thermal outflow in run A.

The outflow collimation is very strong for run $\mathrm{C}$ where $\mathrm{X}$-ray heating is the smallest. The gas is siphoned off within a very narrow channel along the pole. In this case, the fraction of the computational domain occupied by the inflow is the largest. The shape and size of the sonic surface reflects the fact that runs A and B are similar to each other, but they both differ significantly from run C.

To quantify the properties of the inflow and inflow/outflow components, we computed three radial mass rates as a function of radius: (1) the net rate

$$
\dot{M}_{\text {net }}(r)=4 \pi r^{2} \int_{0^{\circ}}^{90^{\circ}} \rho v_{r} \sin \theta d \theta,
$$

(2) the inflow rate

$$
\dot{M}_{\text {in }}(r)=4 \pi r^{2} \int_{0^{\circ}}^{90^{\circ}} \rho v_{r} \sin \theta d \theta \quad \text { for } v_{r}<0,
$$

and (3) the outflow rate

$$
\dot{M}_{\text {out }}(r)=4 \pi r^{2} \int_{0^{\circ}}^{90^{\circ}} \rho v_{r} \sin \theta d \theta \quad \text { for } v_{r}>0 .
$$

We also computed the outflow power carried out in the form of the kinetic energy

$$
P_{\mathrm{k}}(r)=2 \pi r^{2} \int_{0^{\circ}}^{90^{\circ}} \rho v_{r}^{3} \sin \theta d \theta \quad \text { for } v_{r}>0
$$

and in the form of the thermal energy

$$
P_{\mathrm{th}}(r)=4 \pi r^{2} \int_{0^{\circ}}^{90^{\circ}} e v_{r} \sin \theta d \theta \quad \text { for } v_{r}>0
$$

These quantities are shown in Figs. 2, 3, and 4 for run A, $\mathrm{B}$, and $\mathrm{C}$, respectively.

Fig. 2 shows that the outflow in case A is indeed thermal: $P_{\mathrm{k}}<P_{\mathrm{th}}$ for all radii; the outflow starts at radii large compared to $R_{\mathrm{C}}$, its properties are strong functions of radius, i.e., $\dot{M}_{\text {out }}, P_{\mathrm{k}}$, and $P_{\mathrm{th}}$ increase with radius. In particular, almost all the gas inside $r<2 \times 10^{4} r_{*}$ ends up leaving the domain through the inner boundary as there is no or a very weak outflow driven from small radii.

The reduction of $f_{*}\left(=f_{\mathrm{X}}\right)$ compared to $f_{\mathrm{D}}\left(=f_{\mathrm{UV}}\right)$ has a few significant consequences on the outflow properties: in cases B and $\mathrm{C}$ the outflow rate is appreciable even at small radii (see figs. 3 and 4 ). In particular, in case $\mathrm{C}$ the net rate at large radii is almost an order of magnitude smaller than the inflow rate. The latter is almost canceled out by the outflow rate. The overall power of the outflow is $\gtrsim 2$ orders of magnitude higher in cases $\mathrm{B}$ and $\mathrm{C}$ than in case A. The relative weakness of thermal driving for lower $f_{\mathrm{X}}$ cases is obvious when $P_{\mathrm{th}}$ is compared to $P_{\mathrm{k}}$. In run B, $P_{\text {th }}$ increases with increasing radius for $r \lesssim 10^{5}$. But even at its maximum, $P_{\mathrm{th}}$ is 3 orders of magnitude lower than $P_{\mathrm{k}}$. In run C, the kinetic dominance is even greater: $P_{\mathrm{k}}$ is higher than $P_{\text {th }}$ by 4 orders of magnitude.

The dependence of our results on the SED $\left(f_{\mathrm{X}}\right.$ and $\left.f_{\mathrm{UV}}\right)$ and the radiation geometry $\left(f_{*}\right.$ and $\left.f_{\mathrm{D}}\right)$ can be explained by the fact that as $f_{\mathrm{X}}$ and $f_{*}$ decrease the X-ray heating also decreases whereas the radiation flux in the polar region increases compared to the flux at high $\theta$ (see eq. 8). Consequently, an increasing fraction of the inflow from large radii is pushed toward the polar region where radiation pressure exceeds gravity. This compressed inflow is siphoned off by radiation pressure. Perhaps the most intriguing result is that the large scale flow pattern can be very misleading if one wants of assess the relative strength of inflow and flow. Visual inspection of Fig. 1 could lead to the conclusion that the outflow in run $\mathrm{C}$ is much less significant that the outflow in run A because in run $\mathrm{C}$ the outflow is very narrow and barely seen. However, the opposite is true as shown in Table 1. The increase in the mass outflow rate with decreasing $f_{\mathrm{X}}$ is due to increasing $f_{\mathrm{UV}}$ and the increasing efficiency of radiative driving at $f_{\mathrm{X}}$.

Table 1 shows that the inflow rate at $r_{\mathrm{o}}$ increases with decreasing $f_{\mathrm{X}}$. This trend is consistent with accretion theory, in particular with Bondi's estimate for the accretion rate, $\dot{M}_{\mathrm{B}} \propto T_{\infty}^{-3 / 2}$ : weaker X-ray heating results in an increase of the accretion rate because the gravitational sphere of influence over gas pressure is larger. However, a higher $\dot{M}_{\text {min }}$ at large radii does not mean that the inflow rate at small radii must be higher too. We find that the 
mass outflow rate also increases with decreasing $f_{\mathrm{X}}$. Additionally, the efficiency of turning an inflow into an outflow is also sensitive to $f_{\mathrm{X}}:\left|\dot{M}_{\text {out }}\left(r_{\mathrm{o}}\right) / \dot{M}_{\text {in }}\left(r_{\mathrm{o}}\right)\right|=3 / 4,5 / 8$, and $8 / 9$, for $f_{\mathrm{X}}=0.5,0.2$, and 0.05 , respectively. As we mentioned above, the decrease from high to low values of $f_{\mathrm{X}}$ corresponds to a transition from a thermally to radiation driven outflow. We conclude then that thermal driving is less efficient than radiation driving.

One of the parameters specifying our problem is the gas temperature at the outer boundary, $T_{0}$. For the runs A, B, and $\mathrm{C}$, we set the value of $T_{0}$ equal to the Compton temperature. This value of the gas temperature is expected if the gas is optically thin and heating by X-rays from the central object. However, it is possible that the gas at large radii is heated also by other processes (e.g., shocks) or the $\mathrm{X}$-ray heated is reduced by optical depth effects. Therefore, $T_{0}$ can be decoupled from $T_{X}$ and be lower or higher than $T_{r m C}$. To check how are results depend on the assumed value of $T_{0}$, we rerun model $\mathrm{B}$ with $T_{0}$ set to $1 / 10$, $1 / 3$, and 3 of $T_{\mathrm{C}}$, runs B1, B2, and B3, respectively.

As shown in Table 1, $\dot{M}_{\text {in }}\left(r_{\mathrm{o}}\right)$ and $\dot{M}_{\text {net }}\left(r_{\mathrm{i}}\right)$ increase with increasing $T_{0}$. However, one would expect the opposite trend because accretion theory predicts that $\dot{M}_{\mathrm{B}} \propto T_{\infty}^{-3 / 2}$ ( $T_{0}$ is a proxy of $T_{\infty}$ in the simulations). This discrepancy is due to the fact that the above scaling law is derived for the Bondi accretion problem where the gas is adiabatic or isothermal, i.e., where the gas temperature at the critical point increases with $T_{\infty}$. However, in our problem, the gas is not adiabatic as we allow for radiative cooling and heating. This has an important effect on the gas properties (e.g., Zel'dovich \& Novikov 1971). In particular, for $T_{0}>T_{\mathrm{C}}$, radiative processes lead to cooling as the gas accretes. Consequently, the sonic radius is smaller than that for $T_{0}=T_{\mathrm{C}}$. Similarly, for $T_{0}<T_{\mathrm{C}}$, radiative processes lead to heating as the gas accretes. Consequently, the sonic radius is larger than that for $T_{0}=T_{\mathrm{C}}$. Therefore, our results for various $T_{0}$ reflect the basic physical property of accretion flows: flows with a higher temperature at the sonic radius have lower accretion rates than flows with a lower temperature because the gravitational sphere of influence over gas pressure grows with decreasing temperature at the sonic radius.

\section{DISCUSSION AND CONCLUSIONS}

We have calculated a series of models for non-rotating flows which are under the influence of super massive BH gravity and radiation from an accretion disk surrounding the BH. Our numerical approach allows for the selfconsistent determination of whether the flow is gravitationally captured by the $\mathrm{BH}$ or driven away by thermal expansion or radiation pressure. We find that the flow settles quickly into a steady state and has two components (1) an equatorial inflow and (2) a bipolar inflow/outflow with the outflow leaving the system along the pole. The first components is a realization of Bondi-like accretion flow. The second component is an example of a non-radial accretion flow which becomes an outflow once it is pushed close to the rotational axis of the disk where thermal expansion and radiation pressure can accelerate the flow outward. Our main result is that the existence of the above two flow components is robust yet their properties are sensitive to the geometry and SED of the the radiation field and the outer boundaries. In particular, the outflow power and the degree of collimation is higher for the model with the radiation dominated by the UV/disk emission (run C) than for model with the radiation dominated by the X-ray/central engine emission (run A). This sensitivity is related to the fact that thermal expansion compared to radiation pressure drives a weaker, less-collimated outflow.

Our results will likely change if we allow for significant gas rotation. In particular, the gas will tend to converge toward the equator due to the combination of the centrifugal and gravitational forces. This, in turn, will likely weaken the outflow in the polar region because less gas will be pushed toward the polar region. Adding rotation is indeed an important next step. However, within our framework, it requires the introduction of additional free parameters. In particular, we would need to prescribe the rotational rate at the outer boundary. I plan to report on our results from some simulations with rotation in a companion paper.

The treatment of the inner and outer boundaries can affect the results of these simulations. We set up the outer boundary in the spirit of representing steady conditions. However, in real systems but also in our simulations, the conditions at large radii are complex. In particular, we fix the density and internal energy at $r_{\mathrm{o}}$ at all times. This produces self-consistent results for accretion flows but not for outflows because the outflow density and internal energy at $r_{\mathrm{o}}$ do not have to be as those we set there through implementation of the outer boundary conditions. Reruns of our simulations on a larger computational domain and with the outer boundary conditions as predicted by simulations of galaxy evolution such as those performed by Springel, Di Matteo \& Hernquist (2005) would be very important.

It is also important to explore the effects of dust on our results. As we mentioned in $\S 3$, the dust sublimation radius is inside our computational domain. The presence of the dust at large radii can reduce the matter density there, because the radiation pressure on dust can accelerate the matter outward. Consequently, the mass supply rate and density at small radii can decrease. This in turn can lead to higher gas temperature at small radii. If the dust reduces the gas temperature at large radii, based on our simulation with various $T_{0}$, we expect similar changes. In summary, we expect that inclusion of dust will lead to a lower net mass accretion rate and higher temperature at small radii compared to the results presented here.

Our treatment of the inner boundary conditions is one of the simplest possible: free outflow - no matter enters the computational domain through the inner boundary. However, as we mentioned in $\S 1$, quasars have winds which are likely driven from relatively small radii. Therefore, one would need to consider matter entering our computational domain through the inner boundary. Even if the small scale quasar winds do not flow very far from the center, their presence could affect our results. We allow the radiation field from the disk and central object to be attenuated by the gas inside the computational domain. However, PSK showed that disk winds in AGN can be optically thick and they can change the geometry and SED of the disk and central object radiation at large radii, i.e., interior to the inner radius of our computational domain.

We have performed our simulations in the HD limit and 
have not accounted for the effects of magnetic fields. The dynamics of accretion flows can be significantly affected for magnetic fields. In particular, outflows can be magnetically driven from accretion disks (e.g., Blandford \& Payne 1982; Pelletier \& Pudritz 1992 Königl 1993; Emmering, Blandford \& Shlosman 1992; Contopoulos \& Lovelace 1994; Königl \& Kartje 1994; Contopoulos 1995; de Kool \& Begelman 1995; Bottorff et al. 1997; Bottorff, Korista \& Shlosman 2000; Everett, Königl, \& Arav 2002; Proga 2003; Everett 2005). In fact, most magnetohydrodynamic simulations of accretion flows show outflows (e.g., Uchida \& Shibata 1985; Stone \& Norman 1994; Ouyed \& Pudritz 1997; Hawley \& Balbus 2002; Proga \& Begelman 2003b; De Villier et al. 2004; McKinney \& Gammie 2004, and references therein). Additionally, an outflow can be produced even from a flow with angular momentum so low that, if not for the effects of magnetic fields, it would accrete directly onto a $\mathrm{BH}$ without forming a disk (Proga 2005). Thus, magnetic fields can directly and indirectly change our results, e.g., magnetic disk winds, similarly to radiation driven disk winds, can change the SED and they can interact with other flow components. We expect that flow interaction can lead to time-dependent evolution and formation of inhomogeneous inflows and outflows (e.g., Proga \& Begelman 2003b). Therefore, it would be a natural step to repeat simulations similar to ours but in the magnetohydrodynamical limit. We have already set the stage for such a study because simulations performed by Proga (2005) are a close magnetohydrodynamical analog of those presented here. We note that despite two different physical regimes explored here and in our previous work, similarly simple inflow/outflows solutions were obtained. One of the main motivation for this work, is to gain more insights to the general problem of accretion and outflow production where many complex time-dependent flow components are expected. It is also desirable to connect numerical simulations with theoretical predictions such as those obtained by Blandford \& Begelman (1999) and Henriksen \& VallsGabaud (1994).

The SED and consequently gas temperature can be different from those we have in our simulations, even in an optically thin case. One of our simplifications is an assumption that the radiation temperature, $T_{\mathrm{X}}$ (or more generally the SED) does not change with $\theta$. This assumption is motivated by X-ray observations showing that quasar radiation heats a low-density gas, on scales comparable to the Bondi radius, up to an equilibrium Compton temperature of about $2 \times 10^{7} \mathrm{~K}$ (Sazonov et al. 2005; Allen et al. 2006 and references therein). However, the SED and $T_{\mathrm{X}}$ can change with $\theta$. For example, we assume that the $\mathrm{X}$-ray/central object radiation does not change with $\theta$ (see eq. 7) whereas the UV/disk radiation decreases with increasing $\theta$ (see eq. 6). Consequently one could expect the ratio between the X-ray and UV flux to increase with increasing $\theta$. We plan in the near future, to test how important this effect is and try to reconcile the above theoretical expectation with observations.

We assume that a quasar is powered by accretion, but we do not couple the disk accretion rate, that determines radiation, to the mass accretion rate through the inner boundary. This $\dot{M}_{\mathrm{a}}-\dot{M}_{\mathrm{in}}\left(r_{\mathrm{i}}\right)$ decoupling is physically motivated because an accretion disk is build from rotating gas and non-rotating gas will not significantly contribute to the disk formation and disk radiation. In this sense we study radiatively inefficient flows accreting onto an object with a radiatively efficient accretion disk. Additionally, we note that an accretion disk, which is located interior to the inner radius of the computational domain, radiates steadily during our simulations. The fact that the flow in the computational domain settles into a steady state within just a few dynamical time scales shows that our approach is reasonable.

Outflows in our simulations are collimated by the inflowing gas. This collimation can lead to formation of a bottle-neck-like structure as we discovered in run B. There are two sources of concern about the collimation: is it an artifact of the outer boundary or of the numerical resolution. We addressed this issue by rerunning simulation $\mathrm{B}$ and $\mathrm{C}$ with higher resolution in the $\theta$ direction $(100$ grid points instead 50). We found that the results for the higher resolutions are very similar to those with the lower resolution counterparts. The main difference was that the region with $T<10^{5} \mathrm{~K}$ for $r>1.8 \times 10^{5} r_{*}$ is somewhat narrower (by $\sim 1^{\circ}$ ) in the higher resolution run. We also found that the outer boundary are not responsible for the collimation because our test calculations with $r_{\mathrm{o}}$ four times larger than in the runs presented in $\S 3$ show collimation operating in a similar way and far from the outer boundary. Additionally, we note that in run $\mathrm{C}$, collimation begins at $r \sim 5 \times 10^{4} r_{*}$ (i.e., deep inside the computational domain) and is disconnected from the outer boundary, and for that matter, from the inner boundary. To study flow stability, in particular, stability of the outflow, it is important to perform fully three-dimensional simulations which will allow for non-axisymmetric effects and full development of fluid instabilities.

We finish by returning to the questions we raised in $\S 1$. Our simulations show that AGN can have a substantial outflow which originates from the infalling gas. Such an outflow can control the rate at which non-rotating matter is supplied to the AGN central engine as its mass loss rate can be significantly higher than the mass inflow rate at small radii. For example, in run C, as little as $10 \%$ of the inflow at large radii reaches small radii because $90 \%$ of the inflow is turned into an outflow. However, even the power of the strongest outflow is very low compared to the radiation power (i.e., for run $\mathrm{C}, P_{\mathrm{k}} / L=4 \times 10^{-4}$ ). Finally, the gas in our simulations can be related to material responsible for NELs, NALs, and warm absorbers. Additionally, the inflowing gas could be the source of the gas that eventually produces BALs and BELs at radii smaller than the inner radius of our computational domain. We plan to farther explore our model and relax some of our assumptions in order to confirm these conclusions.

ACKNOWLEDGMENTS:

We thank G. Chartas, P. Hopkins, R. Kurosawa, T. Kallman, J. Ostriker, and J. Stone for discussions. We also thank an anonymous referee for comments that help us clarify our presentaion. This work is supported by NASA through grants HST-AR-10305 and HST-AR-10680 from the Space Telescope Science Institute, which is operated by the Association of Universities for Research in Astronomy, Inc., under NASA contract NAS5-26555. Financial support from DoE grant DE-FG52-06NA26217 is also acknowledged. 


\section{REFERENCES}

Allen, S.W., Dunn, R.J.H., Fabian, A.C., Taylor, G.B., Reynolds, C.S. 2006, MNRAS, 372, 21

Antonucci, R.R.J. 1984, ApJ, 278, 499

Awaki, Hisamitsu; Koyama, Katsuji; Inoue, Hajime; Halpern, Jules P. 1991 PASJ, 43, 195

Begelman, M.C., McKee C.F., \& Shields, G.A. 1983, 271, 30

Blanco, Philip R.; Ward, Martin J.; Wright, Gillian S. 1990, MNRAS, 242,4

Blandford R.D., Begelman M.C. 1999, MNRAS, 303, 1

Blandford R.D., Payne D.G. 1982, MNRAS, 199, 883

Blondin, J. M. 1994, ApJ, 435, 756

Bottorff, Korista \& Shlosman 2000

Bottorff, M., Korista, K. T., Shlosman, I., \& Blandford, R. D. 1997, ApJ, 479, 200

Castor, J.I., Abbott, D.C., Klein, R.I. 1975, ApJ, 195, 157

Ciotti L., Ostriker J. P., 1997, ApJ, 487, L105

Ciotti L., Ostriker J. P., 2001, ApJ, 551, 131

Chartas, G., Brandt, W. N., \& Gallagher, S. C. 2003, ApJ, 595, 85

Chelouche, D. \& Netzer, H. 2005, ApJ, 625, 95

Contopoulos, J. \& Lovelace, R.V.E. 1994, ApJ, 429, 139

Contopoulos J. 1995, ApJ, 450, 616

Crenshaw, D. M., Kraemer, S. B., Boggess, A., Maran, S. P., Mushotzky, R. F., \& Wu, C. C. 1999, ApJ, 516, 750

de Kool, M. \& Begelman, M.C. 1995, ApJ, 455, 448

De Villiers, J.-P., Hawley, J. F., Krolik, J. H., \& Hirose, S. 2005, ApJ, 620, 878

Emmering, R.T., Blandford, R.D. \&Shlosman, I. 1992, ApJ, 385, 460

Everett, J.E., Königl, A. \& Arav, N. 2002, ApJ, 569, 671

Everett, J.E. 2005, ApJ, 631, 689

Ferland, G.J., Korista, K.T., Verner, D.A., Ferguson, J.W., Kingdon, J.B., \& Verner, E. M. 1998, PASP, 110, 761

Hamann, F., Barlow, T.A., Cohen, R.D., Junkkarinen, V., \& Burbidge, E.M. 1997, in ASP Conf. Ser. 128, Mass Ejection from Active Galactic Nuclei, eds., N. Arav, I. Shlosman, \& R. Weymann (San Francisco: ASP), p. 19

Hamann, F. \& Ferland, G. 1999, ARA\&A, 37, 487

Hawley, J. F., \& Balbus, S. A. 2002, ApJ, 573, 738

Henriksen, R. N., \& Valls-Gabaud, D. 1994, MNRAS, 266, 681

Hopkins, P.F., Hernquist, L., Cox, T.J., Di Matteo, T., Martini, P., Robertson, B., \& Springel, V. 2005, ApJ, 630, 705

Kaastra, J. S., Mewe, R., Liedahl, D. A., Komossa, S., Brinkman, A. C., 2000, A\&A, 354L, 83

King A., 2003, ApJ, 596, L27

Korista, K.T., Voit, G. M., Morris, S.L., Weymann, R.J. 1993, ApJS, 88,357

Königl A. 1993, in “Astrophysical Jets”, ed. by D.P. O'Dea (Cambridge: Cambridge Univ. Press), 239

Königl, A. \& Kartje, J.F. 1994, ApJ, 434, 446

Kraemer S.B. et al. 2006, ApJ, 633, 693
Krolik, J.H. 1999, Active galactic nuclei: from the central black hole to the galactic environment, Princeton, N.J.: Princeton University Press

Laor, A., Fiore, F., Elvis, E., \& Wilkes B.J., \& McDowell J.C. 1997, ApJ, 477, 93

Laor, A. \& Draine, B.T. 1993, ApJ, 402, 441

McKinney, J. C., \& Gammie, C. F. 2004, ApJ, 611, 977

Murray, N., Chiang, J., Grossman, S.A., \& Voit, G.M. 1995, ApJ, 451, 498 (MCGV)

Murray N., Quataert E., Thompson T. A., 2005, ApJ, 618, 569

Ostriker, E. C., McKee, C.F., \& Klein, R.I. 1991, ApJ, 377, 5930

Ostriker, J.P., Weaver, R., Yahil, A., \& McCray, R. 1976 ApJ, 208, 610

Ouyed R. Pudritz R.E. 1997b, ApJ, 484, 794

Owocki, S. P., Castor, J. I., \& Rybicki, G. B. 1988, ApJ, 335, 914

Pelletier G., Pudritz R.E. 1992, ApJ, 394, 117

Pier, E.A. \& Krolik, J.H. 1992, ApJ, 399, 23

Phinney, E.S. 1989, in Theory of Accretion Disks (NATO ASI Ser. C, 290; Dordrecht: Kluwer), 457

Proga, D. 2005, ApJ, 629, 397

Proga, D., 2007, The Central Engine of Active Galactic Nuclei, ed. L. C. Ho and J.-M. Wang (San Francisco: ASP), in press (astroph0701100)

Proga, D., \& Begelman, M. C. 2003a, 582, 69

Proga, D., \& Begelman, M. C. 2003b, ApJ, 592, 767

Proga, D., \& Kallman, T.R. 2002, ApJ, 565, 455

Proga, D., \& Kallman, T.R. 2004, ApJ, 616, 688

Proga, D., Stone J.M., \& Drew J.E. 1998, MNRAS, 295, 595

Proga, D., Stone J.M., \& Drew J.E. 1999, MNRAS, 310, 476

Proga, D., Stone, J.M., \& Kallman, T.R. 2000, ApJ, 543, 686 (PSK)

Sazonov, S. Y., Ostriker, J. P., Ciotti, L., \& Sunyaev R. A. 2005, MNRAS, 358, 168

Shakura N.I., \& Sunyaev R.A. 1973 A\&A, 24, 337

Shlosman, I, Vitello, P.A. \& Shaviv G. 1985, ApJ, 294, 96

Springel V., Di Matteo T., \& Hernquist L. 2005, ApJ, 620, L79

Stone, J.M., Norman, M.L. 1992, ApJS, 80, 753

Stone, J.M., \& Norman, M.L. 1994, ApJ, 433, 746

Stevens, I. R., \& Kallman, T. R. 1990, ApJ, 365, 321

Turnshek, D. A. 1988, in QSO Absorption Lines: Probing the Universe, ed. J. C. Blades, D. A. Turnshek, \& C. A. Norman (Cambridge: Cambridge Univ. Press), 17

Uchida Y., Shibata K. 1985, PASJ, 37, 515

Wang, J.-M., Chen, Y.-M.; Hu, C. 2006, ApJ, 637, L85

Wittkowski, M.; Kervella, P.; Arsenault, R.; Paresce, F.; Beckert, T.; Weigelt, G. 2004, A\&A, 418, L39

Woods, D.T., Klein, R.I., Castor, J.I., McKee, C.F., \& Bell, J.B. 1996, ApJ, 461, 767

Zel'dovich, Ya. B., \& Novikov, I. D. 1971, Relativistic Astrophysics. I. Stars and Relativity (Chicago: Univ. Chicago Press)

Zheng, W., Kriss, G.A., Telfer, R.C., Grimes, J.P., \& Davidsen, A.F. 1997, ApJ, 475, 469 
TABLE 1

Summary OF RESUlts

\begin{tabular}{|c|c|c|c|c|c|c|c|c|c|c|c|}
\hline Run & $f_{\mathrm{D}}$ & $f_{*}$ & $f_{\mathrm{UV}}$ & $f_{\mathrm{X}}$ & $\begin{array}{c}T_{0} \\
2 \times 10^{7} \mathrm{~K}\end{array}$ & $\begin{array}{c}\dot{M}_{\mathrm{in}}\left(r_{\mathrm{o}}\right) \\
10^{25} \mathrm{~g} \mathrm{~s}^{-1}\end{array}$ & $\begin{array}{c}\dot{M}_{\text {net }}\left(r_{\mathrm{i}}\right) \\
10^{25} \mathrm{~g} \mathrm{~s}^{-1}\end{array}$ & $\begin{array}{c}\dot{M}_{\text {out }}\left(r_{\mathrm{o}}\right) \\
10^{25} \mathrm{~g} \mathrm{~s}^{-1}\end{array}$ & $\begin{array}{c}v_{r} \\
\mathrm{~km} \mathrm{~s}^{-1}\end{array}$ & $\begin{array}{c}P_{\mathrm{k}}\left(\mathrm{r}_{o}\right) \\
10^{40} \mathrm{erg} \mathrm{s}^{-1}\end{array}$ & $\begin{array}{c}P_{\mathrm{th}}\left(\mathrm{r}_{o}\right) \\
10^{40} \mathrm{erg} \mathrm{s}^{-1}\end{array}$ \\
\hline A & 0.5 & 0.5 & 0.5 & 0.5 & 1 & -4 & -1 & 3 & 700 & 2 & 4 \\
\hline B & 0.8 & 0.2 & 0.8 & 0.2 & 1 & -8 & -3 & 5 & 4000 & 100 & 0.8 \\
\hline B1 & 0.8 & 0.2 & 0.8 & 0.2 & $1 / 10$ & -0.5 & -0.09 & 0.41 & 1500 & 0.5 & 0.8 \\
\hline B2 & 0.8 & 0.2 & 0.8 & 0.2 & $1 / 3$ & -2 & -0.4 & 1.6 & 1700 & 2 & 2 \\
\hline B3 & 0.8 & 0.2 & 0.8 & 0.2 & 3 & -9 & -5 & 4 & 400 & 3 & 0.8 \\
\hline C & 0.95 & 0.05 & 0.95 & 0.05 & 1 & -9 & -1 & 8 & 6700 & 300 & 0.03 \\
\hline
\end{tabular}



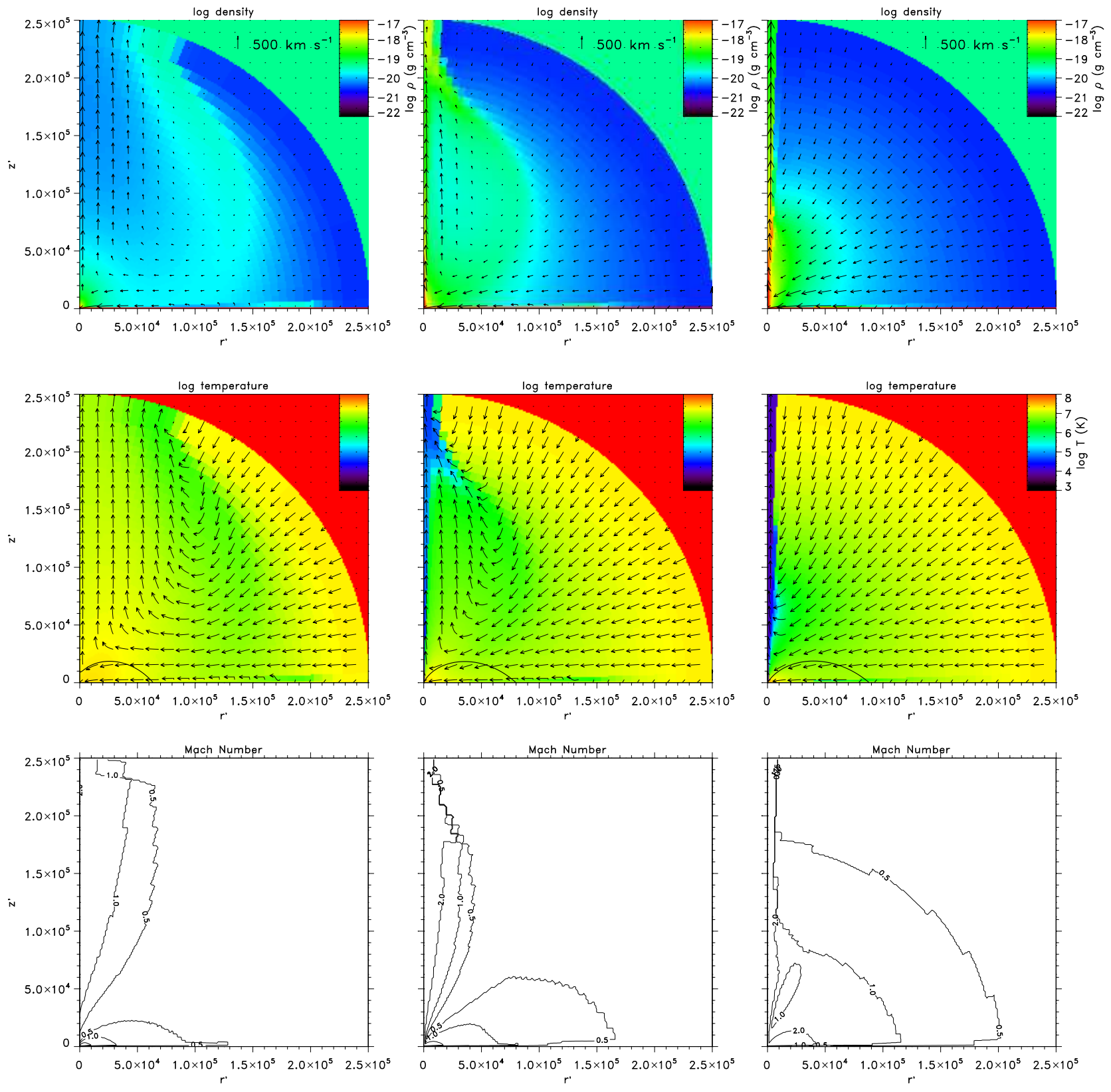

FIG. 1.- Comparison of the results for run A, B, and C (left, middle, and right column, respectively). Top row of panels: Maps of logarithmic density overplotted by the poloidal velocity. For clarity, the arrows are plotted with the maximum velocity set to $1000 \mathrm{~km} \mathrm{~s}{ }^{-1}$. Middle row of panels: Maps of logarithmic temperature overplotted by the direction of the poloidal velocity. The solid curve in the bottom left corner marks the position of the Compton radius corrected for the effects of radiation pressure due to electron scattering (see eq. 19 in the main text). Bottom row of panels: Contours of the Mach number. The length scale is in units of the inner disk radius (i.e., $r^{\prime}=r / r_{*}$ and $\left.z^{\prime}=z / r_{*}\right)$. 

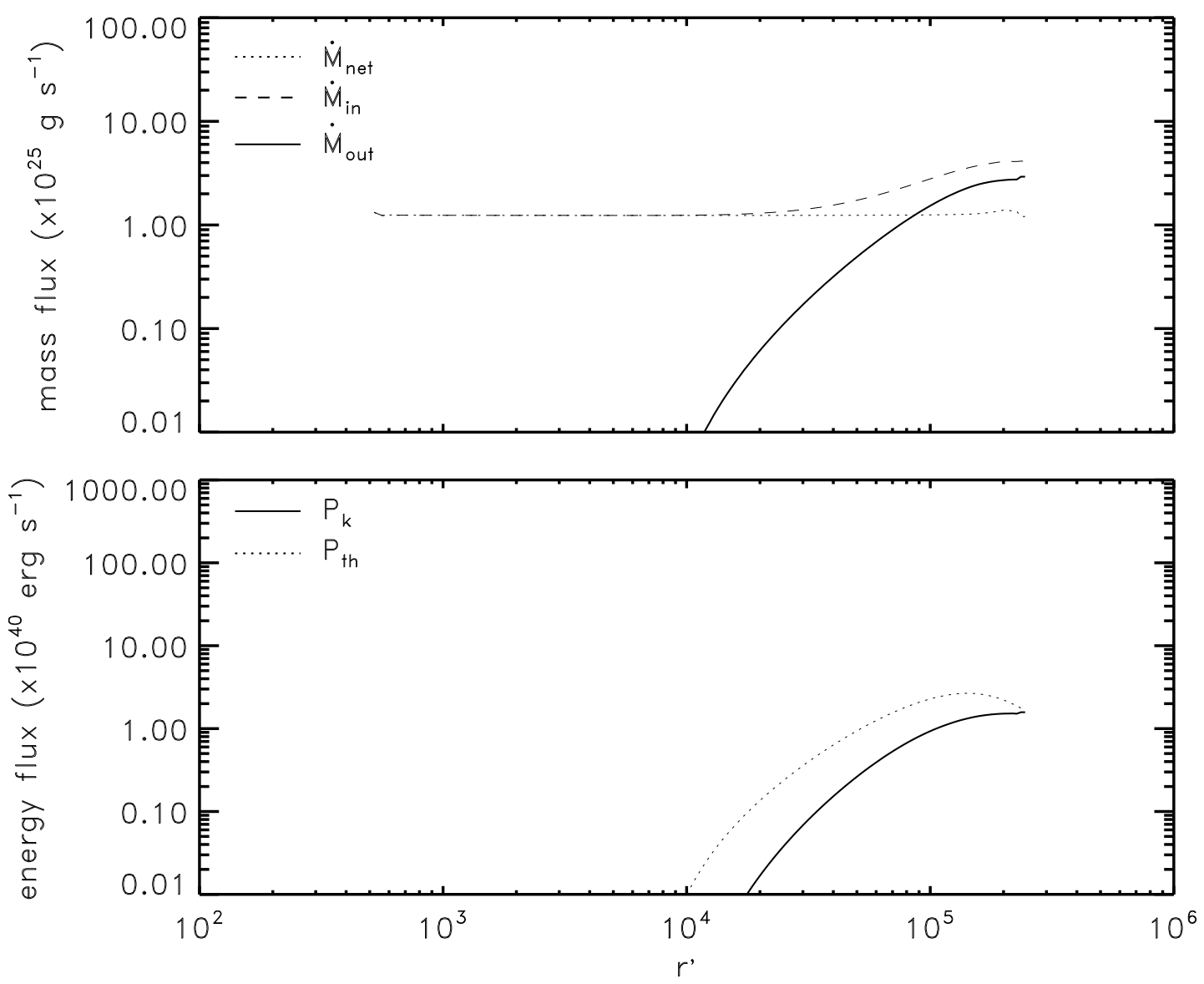

FIG. 2.- Top panel: The mass flux rates as a function of radius for run A. The solid, dashed, and dotted line corresponds to the outflow, inflow, and net rates, respectively (see eqs. 20, 21, and 22 for the formal definitions). Note that the absolution value of the inflow and net rates are plotted because these quantities are negative. Bottom panel: The energy fluxes carried out by the outflow as a function of radius in run A1. The solid and dashed line corresponds to the kinetic and thermal energy flux, respectively (see eqs. 23 and 24 for the formal definitions). The length scale is in units of the inner disk radius (i.e., $r^{\prime}=r / r_{*}$ ). 

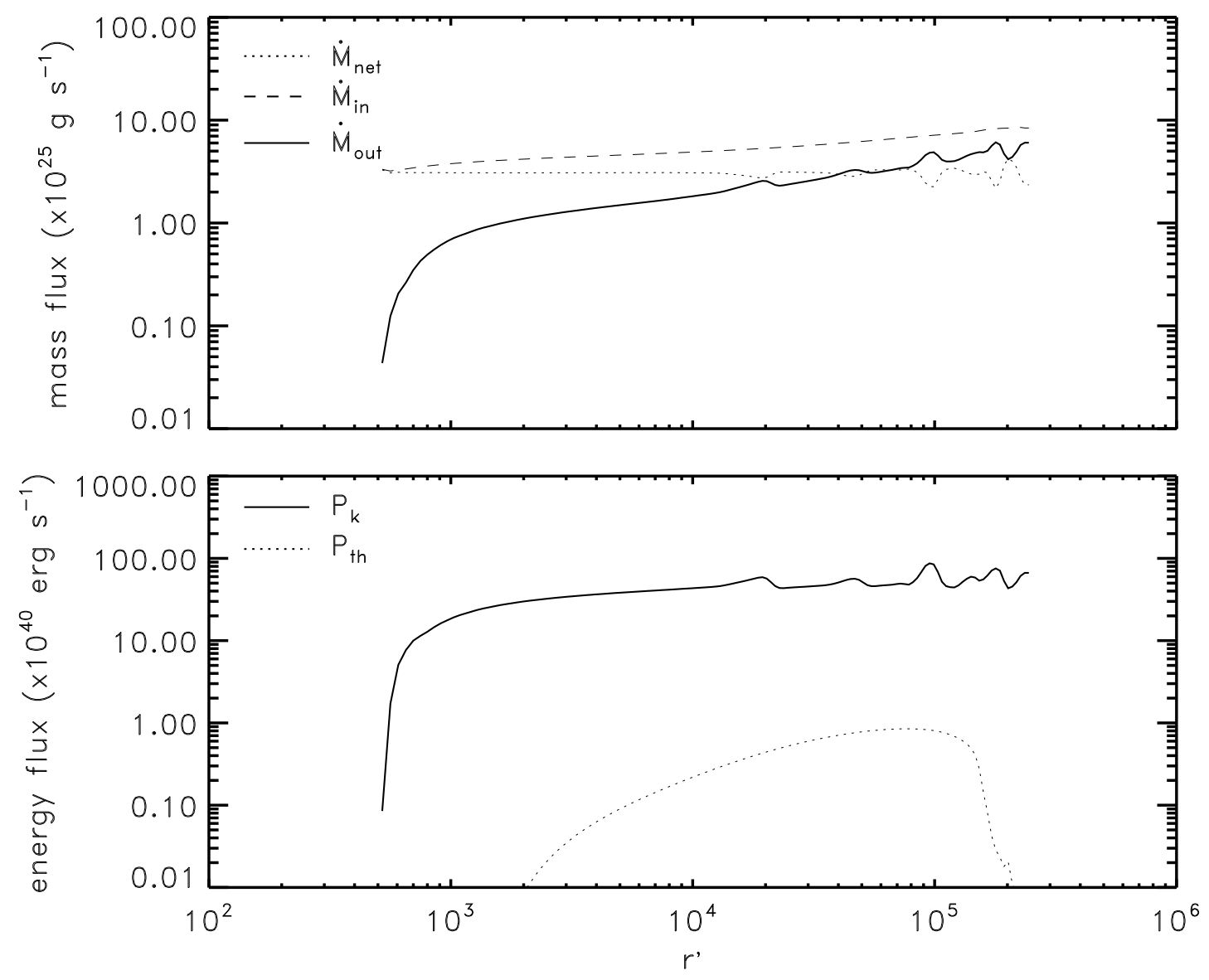

FIG. 3.- As Fig. 2 but for run B. 

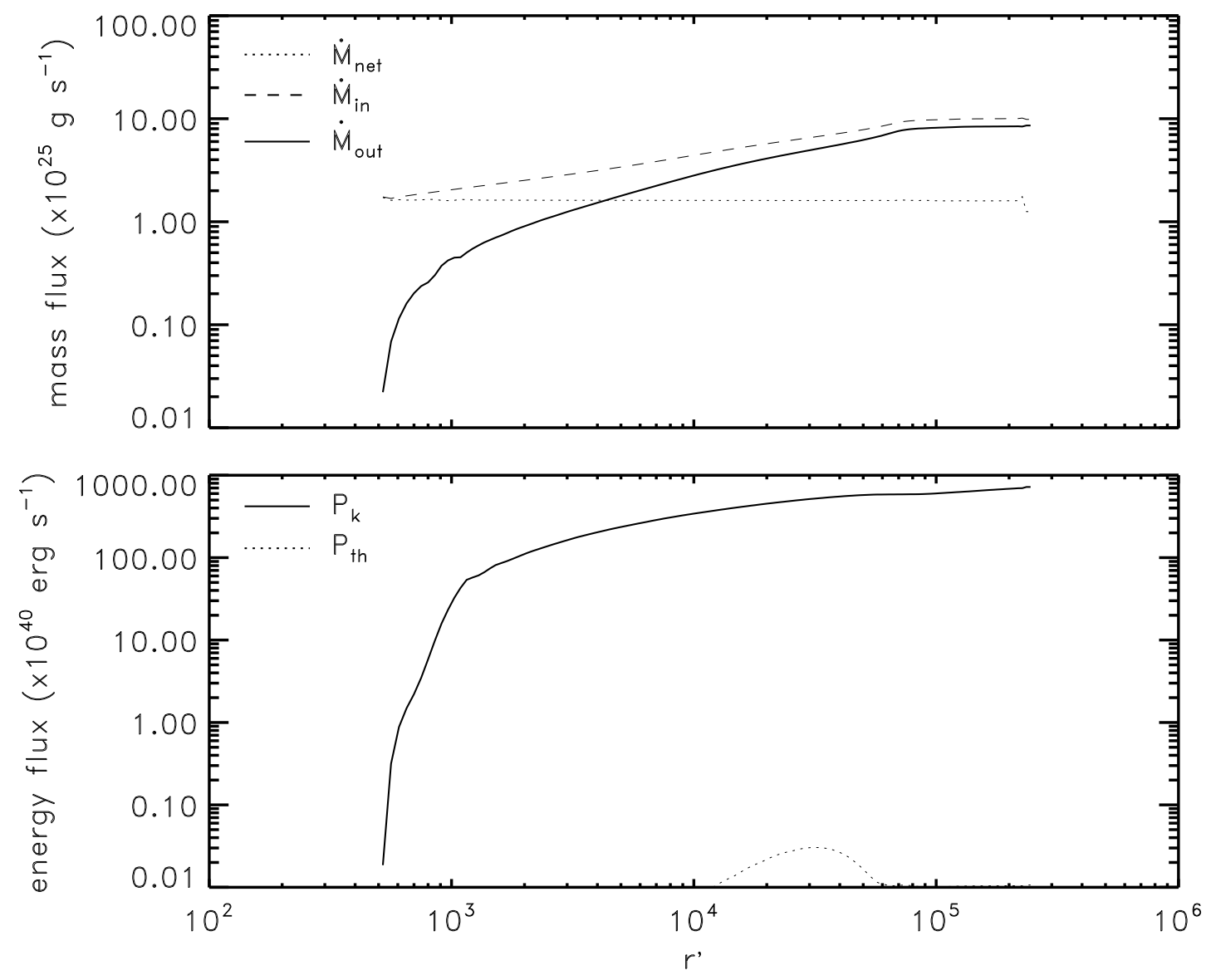

FIG. 4.- As Fig. 2 but for run C. 